\title{
Clinicopathological Analysis of Patients with Nonmuscle-Invasive Bladder Cancer Who Underwent Radical Cystectomy
}

\author{
Naotaka Nishiyama ${ }^{1,2^{*}}$, Hiroshi Kitamura ${ }^{2}$, Masatoshi Mutoh ${ }^{1}$, Masahiro Yanase ${ }^{1}$, Hiroyuki Iwaki ${ }^{3}$, \\ Tadashi Hasegawa $^{4}$, Taiji Tsukamoto ${ }^{2}$ \\ ${ }^{1}$ Divisions of Urology, Sunagawa City Medical Center, Sunagawa, Japan \\ ${ }^{2}$ Departments of Urology, Sapporo Medical University School of Medicine, Sapporo, Japan \\ ${ }^{3}$ Divisions of Pathology, Sunagawa City Medical Center, Sunagawa, Japan \\ ${ }^{4}$ Departments of Surgical Pathology, Sapporo Medical University School of Medicine, Sapporo, Japan \\ Email: nishiyan@sapmed.ac.jp
}

Received May 26, 2012; revised June 16, 2012; accepted June 26, 2012

\begin{abstract}
Objectives: Surgical specimens obtained at the time of the last transurethral resection of bladder tumor (TURBT) for patients with nonmuscle-invasive bladder cancer (NMIBC) who underwent radical cystectomy were retrospectively evaluated in order to investigate the relationship between pathological variation and upstaging of NMIBC. Methods and Materials: Twenty patients (19 men, 1 woman; aged $69.4 \pm 12.1$ (mean \pm SD) years) diagnosed with NMIBC underwent radical cystectomy during follow-up. Results: Five of the 20 patients $(25 \%)$ had pathological upstaging in the radical cystectomy specimens. There was a statistical association between pathological upstaging and cancer death $(p=$ 0.002). There were three patterns of pathological variation in the upstaged specimens: 1) In patients with BCG-resistant NMIBC, urothelial carcinoma invaded through the lamina propria; 2) Urothelial carcinoma showed diffuse invasion beyond the deep lamina propria, and the cancer cells had infiltrated as single cells and formed nodules; 3) TURBT specimens showed a micropapillary variant. Conclusions: Since these pathological variations correlated with pathological upstaging, they may provide an indication for cystectomy in NMIBC patients.
\end{abstract}

Keywords: Clinicopathological Examination; Nonmuscle-Invasive Bladder Cancer; Radical Cystectomy; Pathological Upstaging

\section{Introduction}

Approximately $70 \%-75 \%$ of bladder tumors are nonmuscle-invasive bladder cancer (NMIBC) confined to the mucosal and submucosal layers (stage Ta, Tis, T1) of the bladder at the time of initial evaluation. The 5-year cause-specific survival rate for NMIBC is high and it is curable by transurethral intervention, although the intravesical recurrence rate is higher than $50 \%$ [1-5]. However, high-risk NMIBC — having a high histological grade and lamina propria invasion-has a poor clinical outcome. High-risk NMIBC is associated with progression, metastasis, and cancer death, with between $30 \%-50 \%$ of patients having progressive disease. The 5-year causespecific survival after radical cystectomy is $40 \%-80 \%$ [6-10]. To decrease the recurrence rate and improve causespecific survival, early cystectomy is recommended for high-risk NMIBC $[9,10]$. However, radical cystectomy, despite advances in urinary diversion techniques, has its

${ }^{*}$ Corresponding author. disadvantages: it impairs quality of life, some patients are overtreated with this aggressive therapy, and the morbidity and mortality are $20 \%$ and $1 \%-4 \%$, respectively [8].

It is thus important to restrict the criteria for radical cystectomy to high-risk NMIBC. Several studies have shown that the heterogeneous biological behavior of stage T1 NMIBC is related to depth of invasion beyond the lamina propria [11-18]. Although there is a correlation between depth of invasion within the lamina propria and prognosis, these classifications have not gained formal acceptance. In this study, surgical specimens from transurethral resection of bladder tumor (TURBT) and cystectomy for NMIBC patients were evaluated in order to investigate the relationship between pathological variations and upstaging of NMIBC.

\section{Patients and Methods}

\subsection{Patients and Tissue Samples (Tables 1 and 2)}

Between January 1998 and November 2008, 138 patients 
Table 1. Patients characteristics.

\begin{tabular}{|c|c|c|}
\hline Characteristic & & No. \\
\hline Age & & $69.4 \pm 12.1$ \\
\hline \multirow[t]{2}{*}{ Sex } & Male & $19(95 \%)$ \\
\hline & Female & $1(5 \%)$ \\
\hline Average number of NMIBC recurrences & & $1.7(0-8)$ \\
\hline \multirow{2}{*}{ Grade $^{*}$} & G1-2 & $7(35 \%)$ \\
\hline & G3 & $13(65 \%)$ \\
\hline \multirow{3}{*}{ Depth of invasion ${ }^{*}$} & Tis & $6(30 \%)$ \\
\hline & $\mathrm{Ta}$ & $1(5 \%)$ \\
\hline & $\mathrm{T} 1$ & $13(65 \%)$ \\
\hline \multirow[t]{2}{*}{ Carcinoma in situ } & Present & $13(65 \%)$ \\
\hline & Not present & $7(35 \%)$ \\
\hline \multirow{2}{*}{ Macroscopic organization ${ }^{*}$} & Papillary & $9(45 \%)$ \\
\hline & Non-papillary & $11(55 \%)$ \\
\hline \multirow[t]{2}{*}{ BCG treatment } & Performed & $8(40 \%)$ \\
\hline & Not performed & $12(60 \%)$ \\
\hline
\end{tabular}

*At time of last transurethral resection; ${ }^{\$}$ Transformation from superficial papillary carcinoma to non-papillary invasive carcinoma is included in the "nonpapillary" macroscopic organization. were diagnosed with NMIBC (stage Ta, T1 or Tis) at the Sunagawa City Medical Center, Sunagawa, Japan. All these patients underwent TURBT as initial treatment. Usually, we confirmed the condition of the muscularis propria as far as was possible at the time of TURBT. Twenty of those patients whose TURBT specimens did not reveal muscle invasion of the bladder (19 men, 1 woman; aged $69.4 \pm 12.1$ (mean $\pm \mathrm{SD}$ ) years) underwent radical cystectomy during follow-up. Patients who had had upper urinary tract disease were excluded. Eleven of these 20 patients underwent radical cystectomy after the initial TURBT. The remaining patients had this radical surgery after recurrence and intravesical treatment showed no muscle invasion. Because the TURBT specimens showed that these patients were at high risk for progression, we performed radical cystectomy without a second TURBT. They underwent radical cystectomy because transurethral resection could not control NMIBC due to tumor multiplicity or multiple intravesical recurrences after intravesical instillation of bacillus Calmette-Guerin (BCG), anti-cancer drugs, or both.

All TURBT and cystectomy specimens were reviewed

Table 2. Clinicopathological characteristics and outcomes of patients who underwent radical cystectomy for NMIBC.

\begin{tabular}{|c|c|c|c|c|c|c|c|}
\hline \multirow[b]{2}{*}{ Case } & \multirow[b]{2}{*}{ Age } & \multirow[b]{2}{*}{ Sex } & \multirow{2}{*}{$\begin{array}{c}\text { Depth of invasion } \\
\text { At time of } \\
\text { last TURBT }\end{array}$} & \multirow{2}{*}{$\begin{array}{c}\text { Depth of invasion } \\
\begin{array}{c}\text { At time of } \\
\text { cystectomy }\end{array}\end{array}$} & \multirow[b]{2}{*}{ Micropapillary variant } & \multirow[b]{2}{*}{ Lymph node metastasis at time of cystectomy } & \multirow[b]{2}{*}{ Prognosis } \\
\hline & & & & & & & \\
\hline $\mathrm{T} 1$ & 80 & Male & Tis & T0 & - & - & alive \\
\hline $\mathrm{T} 2$ & 83 & Male & Ta & T0 & - & - & alive \\
\hline $\mathrm{T} 3$ & 48 & Male & $\mathrm{T} 1$ & Tis & - & - & alive \\
\hline $\mathrm{T} 4$ & 61 & Male & $\mathrm{T} 1$ & T0 & - & - & alive \\
\hline T5 & 72 & Male & Tis & Tis & - & - & alive \\
\hline T6 & 61 & Male & $\mathrm{T} 1$ & T0 & - & - & alive \\
\hline $\mathrm{T} 7$ & 78 & Male & $\mathrm{T} 1$ & $\mathrm{~T} 1$ & - & - & death: not UC \\
\hline $\mathrm{T} 8$ & 58 & Male & $\mathrm{T} 1$ & $\mathrm{~T} 1$ & - & - & alive \\
\hline T9 & 72 & Male & $\mathrm{T} 1$ & $\mathrm{~T} 1$ & - & - & alive \\
\hline $\mathrm{T} 10$ & 76 & Male & $\mathrm{T} 1$ & $\mathrm{~T} 1$ & - & - & alive \\
\hline $\mathrm{T} 11$ & 42 & Male & $\mathrm{T} 1$ & $\mathrm{~T} 1$ & - & - & alive \\
\hline $\mathrm{T} 12$ & 78 & Male & $\mathrm{T} 1$ & Tis & - & - & alive \\
\hline $\mathrm{T} 13$ & 80 & Male & Tis & Tis & - & - & alive \\
\hline $\mathrm{T} 14$ & 75 & Male & Tis & Tis & - & - & alive \\
\hline $\mathrm{T} 15$ & 75 & Male & $\mathrm{T} 1$ & $\mathrm{~T} 1$ & - & - & alive \\
\hline $\mathrm{T} 16$ & 83 & Female & Tis & $\mathrm{T} 3 \mathrm{~b}$ & - & - & cancer death \\
\hline $\mathrm{T} 17$ & 81 & Male & Tis & $\mathrm{T} 3 \mathrm{~b}$ & - & - & alive \\
\hline $\mathrm{T} 18$ & 52 & Male & $\mathrm{T} 1$ & $\mathrm{~T} 2 \mathrm{a}$ & - & - & alive \\
\hline $\mathrm{T} 19$ & 68 & Male & $\mathrm{T} 1$ & T3a & - & - & cancer death \\
\hline $\mathrm{T} 20$ & 65 & Male & $\mathrm{T} 1$ & $\mathrm{~T} 1$ & + & + & cancer death \\
\hline
\end{tabular}

${ }^{1}$ Transurethral resection of bladder cancer. -: negative; +: positive. 
by two pathologists independently. The pathological variations of urothelial carcinoma (UC) invading the lamina propria was analyzed in the TURBT specimens. Those patients whose TURBT specimens revealed invasion through the muscularis propria were excluded. Among all specimens at the time of the last TURBT, 7 and 13 were graded as Grade1-2 (G1-2) and Grade3 (G3) $\mathrm{UC}$, respectively, on the basis of the 1973 WHO classification system, and 6, 1, and 13 were classed as Tis, Ta, and $\mathrm{T} 1$, respectively according to the TNM system. Pathological upstaging was defined as UC with muscularis propria invasion or lymph node metastasis in the cystectomy specimens.

\subsection{Statistics}

Correlations between the prognosis and pathological upstaging of NMIBC were analyzed using the unpaired $t$ test. Differences at $p<0.05$ were considered significant.

\section{Results}

Twenty patients underwent radical cystectomy for NMIBC based on the pathological diagnosis in TURBT specimens. The mean follow-up interval from the first diagno- sis was $57.8 \pm 29$ months (mean $\pm \mathrm{SD}$ ). The reasons for radical cystectomy were intravesical recurrence after BCG therapy in 8 patients, multiple and large tumors that were difficult to control by TURBT in 6 patients, and a high risk of progression as classified by the EAU guidelines in 6 patients (http://www.uroweb.org/gls/pdf/05_Ta T1_Bladder_Cancer.pdf). Five of the 20 patients $(25 \%)$ had pathological upstaging to MIBC in which UC invaded through the muscularis propria at the time of cystectomy; the remaining patients had no pathological upstaging. Three of the 5 upstaged patients died due to UC, whereas none of 15 patients without upstaging died of UC (Table 2). There was a significant association between pathological upstaging and cancer death $(p=$ 0.002).

Figure 1 shows pathological specimens from last TURBTs and cystectomies. Three pathological patterns were identified in the specimens from patients with upstaging: macroscopically flat UC invasion after BCG therapy, nodular and single-cell infiltration, and a micropapillary variant. In the first pattern, the cystectomy specimens revealed that macroscopically flat UC had invaded beyond the perivesical tissue but could not be recognized as exophytic tumor lesions. However, microscopically at
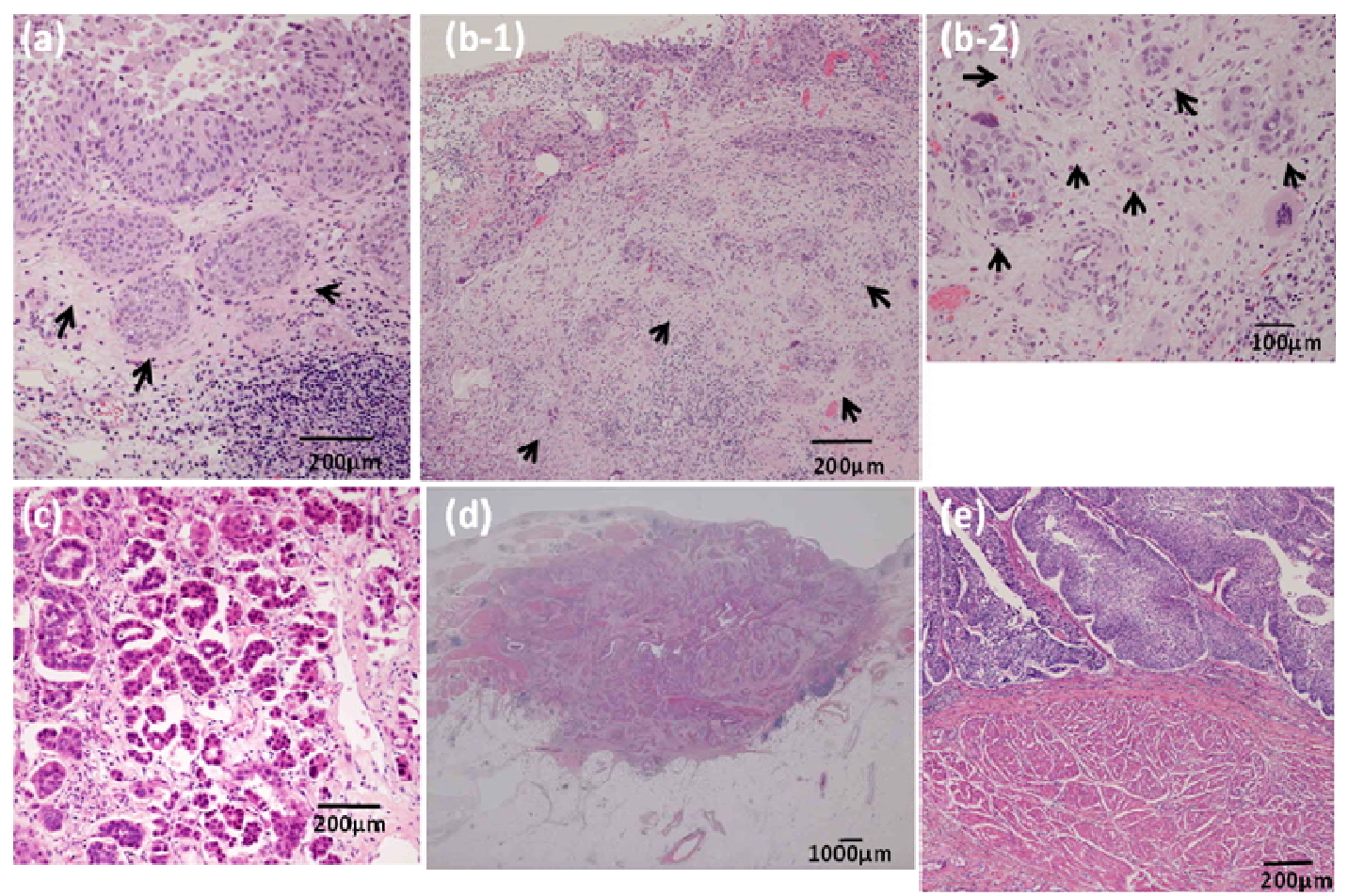

Figure 1. Pathological specimens at the time of last TURBT and cystectomy. (a) After BCG therapy, urothelial carcinoma invades through the lamina propria above the muscularis mucosa or minimal nodular urothelial carcinoma invades through the lamina propria at the time of the last TURBT (arrows); (b) Urothelial carcinoma forms diffuse invasion beyond the deep lamina propria at the time of last TURBT, forming nodules and infiltrating as single cells (arrows); (c) TURBT specimens show the micropapillary variant; (d) After BCG treatment, urothelial carcinoma invades through the perivesical tissue, which could not be recognized as tumor morphology in the cystectomy specimens; (e) Urothelial carcinoma invades through the lamina propria compressively near the muscularis propria. 
the last TURBT, UC had invaded through the lamina propria but not through the muscularis propria, or minimal nodular UC had invaded through the lamina propria in 2 patients (T16-17) who had intravesical recurrence after BCG therapy (Figures 1(a) and (d)). In the second pattern, the cystectomy specimens revealed that UC had invaded diffusely through the muscularis propria. In the last TURBT specimens, UC had invaded beyond the deep lamina propria. These cancer cells caused nodular lesions and single-cell infiltrations. These 2 patients (T18-19) had no history of intravesical BCG treatment (Figure 1(b)). In the last upstaged pattern, the cystectomy and TURBT specimens revealed a micropapillary variant in one patient (T20) (Figure 1(c)). In the cystectomy specimens, the micropapillary variant was mainly seen as lymphovascular invasion.

We showed that there was pathological variation without upstaging of patients in the case of UC that invaded through the lamina propria but not through the muscularis mucosa or only a slightly nodular lamina propria. All had a history of intravesical BCG treatment (T1-6). UC invaded the lamina propria compressively near the muscularis propria (Figure 1(e)) (T7-12). The specimens revealed many UC cancer nests invading through the lamina propria, spreading carcinoma in situ, or both (image not shown) (T13-15).

\section{Discussion}

In this study, 5 of the 20 patients (25\%) who underwent radical cystectomy for NMIBC had pathological upstaging in which UC invaded through the muscularis propria at the time of radical cystectomy. Three of the 5 upstaged patients died due to urothelial carcinoma, whereas no patient without upstaging died of UC. A significant association was found between pathological upstaging and cancer death $(p=0.002)$.

Fritsche et al. reported that, for patients with high grade $\mathrm{T} 1$ bladder cancer, $51.4 \%$ were upstaged to the muscle-invasive stage (T2 or higher), and $33.4 \%$ were upstaged to the non-organ-confined stage during the follow-up period [6]. In the present study, 3 (23\%) of 13 patients who were diagnosed with $\mathrm{T} 1$ bladder cancer at TURBT were upstaged to MIBC or lymph node metastasis. The upstaging rate of $\mathrm{T} 1$ bladder cancer was therefore lower than that reported by Fritshe et al., validating our indication for radical cystectomy in patients with $\mathrm{T} 1$ bladder cancer.

To improve the survival of high-risk NMIBC patients, early cystectomy is one option. Herr et al. reported that, for NMIBC patients with relapse, $92 \%$ survived when cystectomy was performed within 2 years, compared with $56 \%$ who underwent cystectomy after 2 years of follow-up. Of the patients with relapsed muscle-invasive cancer, $41 \%$ survived after early cystectomy versus $18 \%$ after delayed cystectomy [9]. Other studies reported cancer-specific survival rates of NMIBC patients with immediate cystectomy of $70 \%-90 \%$ compared to about $50 \%$ for delayed cystectomy [6-8]. In our study, even though 9 of the $13 \mathrm{~T} 1$ bladder cancer patients underwent immediate cystectomy, 2 died of bladder cancer. These 2 patients had nodular and infiltrative invasion beyond the deep lamina propria and exhibited the micropapillary variant. In contrast, of the patients who were not upstaged, none died of urothelial carcinoma in this study. Although early cystectomy may improve the survival of high-risk NMIBC patients, cystectomy also has potential disadvantages. The perioperative mortality and morbidity rates are not trivial, being $1 \%-6 \%$ and $30 \%$, respectively. Moreover, patients who have undergone cystectomy may have long-term changes in sexual, gastrointestinal, and genitourinary functions. Thus, cystectomy might be considered overtreatment in some cases and bladder preservation may be indicated [7]. Therefore, the indication of radical cystectomy for patients with high grade T1 disease remains controversial.

In terms of the association found between pathological variations and upstaging, pathological review at the time of the last TURBT showed three patterns of lamina propria invasion (Figures 1(a)-(c)). For patients who received intravesical $B C G$, pathological review of the TURBT specimens of both upstaged and non-upstaged patients showed similar lamina propria invasion. In the cystectomy specimens of the upstaged patients, the fact that macroscopically flat UC invading beyond the perivesical tissue could not be recognized as a tumor lesion indicates the difficulties of performing complete resection of BCG-refractory NMIBC by TURBT as well as distinguishing the pathology of BCG-refractory NMI$\mathrm{BC}$ with upstaging from that without upstaging from TURBT specimens only. Brake et al. reported muscleinvasive progression in $58 \%$ of patients with recurrence after BCG [11]. In addition, a 71\% cumulative incidence of progression to muscle invasion at 5 years $(48 \%$ death from disease) was noted for patients treated with a second course of BCG [7]. Therefore, if we confirm that $\mathrm{UC}$ invades through the lamina propria after BCG treatment, radical cystectomy should be the first choice of treatment.

In this study, we showed that upstaged patients had diffuse invasion through the deep lamina propria at the time of TURBT. These cancer cells caused nodular lesions and single-cell infiltrations. These pathologies were not seen in patients without upstaging. Several reports have suggested that there is a strong correlation between depth of invasion and prognosis [11-18]. Cheng et al. proposed substaging NMIBC based on the depth of invasion measured using a micrometer. The 5-year progression-free 
survival rate for patients with depth of invasion of $<1.5$ mm was $93 \%$, compared with $67 \%$ for those with depth of invasion of $\geq 1.5 \mathrm{~mm}[12,13]$. Jimenez et al. reported that the presence of any infiltrative pattern in UC led to a worse prognosis than for those without an infiltrative pattern [19]. Therefore, if both deep lamina propria invasion and cancer having a nodular pattern or single cell infiltrates are present, these NMIBCs will have a higher frequency of upstaging and a poorer prognosis.

Micropapillary urothelial carcinoma is a rare variant. The first histological subtype is composed of filiform projections lacking a vascular core. The second histological subtype is composed of clusters of cells showing reverse polarity. These clusters are surrounded by clear spaces or retraction artifacts that separate them from the surrounding stroma. In cancers with this component, $56 \%$ metastasized [20]. Kamat et al. reported that, of patients having nonmuscle-invasive micropapillary UC treated with BCG therapy, $67 \%$ developed disease progression, including $22 \%$ who developed metastatic disease. Compared to patients treated with initial cystectomy, those who underwent the procedure after progression had higher incidences of non-organ-confined disease and node-positive disease $[21,22]$. In our patients with micropapillary UC, cystectomy specimens revealed lymph node metastasis, but no muscle invasion. Micropapillary UC is typically aggressive and presents with advanced-stage disease in most cases. Due to the high frequency of metastasis, NMIBC with micropapillary components should be treated by radical cystectomy.

\section{Conclusions}

Our results suggest that pathological evaluation of specimens from TURBT is important to decide if radical cystectomy is indicated in patients with high-risk NMIBC. However, the limitation in this study was its retrospective nature and small patient population. Moreover, the patients in our study did not undergo a second TURBT. Thus, further prospective studies that include many cases and routine second TURBT are needed to ascertain the validity of our conclusions.

This work was supported in part by a Grant-in-Aid for Clinical Cancer Research (H22-Clinical Cancer-026, Project ID: 10103787) from the Ministry of Health, Labor and Welfare of Japan.

\section{REFERENCES}

[1] D. J. Parekh, B. H. Bochner and G. Dalbagni, "Superficial and Muscle-Invasive Bladder Cancer: Principles of Management for Outcomes Assessments," Journal of Clinical Oncology, Vol. 24, No. 35, 2006, pp. 19-27. doi:10.1200/JCO.2006.08.5431

[2] H. Kitamura and T. Tsukamoto, "Early Bladder Cancer:
Concept, Diagnosis, and Management," International Journal of Clinical Oncology, Vol. 11, No. 1, 2006, pp. 28-37. doi:10.1007/s10147-006-0552-y

[3] E. Okajima, H. Fujimoto, Y. Mizutani, Y. Mizutani, E. Kikuchi, H. Koga, S. Hinotsu, N. Shinohara and T. Miki, "Cancer Death from Non-Muscle Invasive Bladder Cancer: Report of the Japanese Urological Association of Data from the 1999-2001 Registry in Japan," International Journal of Urology, Vol. 17, No. 11, 2010, pp. 905-912. doi:10.1111/j.1442-2042.2010.02633.x

[4] A. Lopez-Beltran, "Bladder Cancer: Clinical and Pathological Profile," Scandinavian Journal of Urology and Nephrology, Vol. 42, No. 4, 2008, pp. 95-109.

[5] K. Matsumoto, E. Kikuchi, Y. Horiguchi, N. Tanaka, A. Miyajima, K. Nakagawa, J. Nakashima and M. Oya, "Late Recurrence and Progression in Non-Muscle-Invasive Bladder Cancers after 5-Year Tumor-Free Periods," Urology, Vol. 75, No. 6, 2010, pp. 1385-1390.

doi:10.1016/j.urology.2009.09.088

[6] H. M. Fritsche, M. Burger, R. S. Svatek, C. Jeldres, P. I. Karakiewicz, G. Novara, E. Skinner, S. Denzinger, Y. Fradet, H. Isbarn, P. J. Bastian, B. G. Volkmer, F. Montorsi, W. Kassouf, D. Tilki, W. Otto, U. Capitanio, J. I. Izawa, V. Ficarra, S. Lerner, A. I. Sagalowsky, M. Schoenberg, A. Kamat, C. P. Dinney, Y. Lotan and S. F. Shariat, "Characteristics and Outcomes of Patients with Clinical T1 Grade 3 Urothelial Carcinoma Treated with Radical Cystectomy: Results from an International Cohort," European Urology, Vol. 57, No. 2, 2010, pp. 300-309. doi:10.1016/j.eururo.2009.09.024

[7] G. S. Kulkarni, O. W. Hakenberg, J. E. Gschwend, G. Thalmann, W. Kassouf, A. Kamat and A. Zlotta, “An Updated Critical Analysis of the Treatment Strategy for Newly Diagnosed High-Grade T1 (Previously T1G3) Bladder Cancer," European Urology, Vol. 57, No. 1, 2010, pp. 60-70. doi:10.1016/j.eururo.2009.08.024

[8] M. S. Soloway, M. Sofer and A. Vaidya, "Contemporary Management of Stage T1 Transitional Cell Carcinoma of the Bladder," Journal of Urology, Vol. 167, No. 4, 2002, pp. 1573-1583. doi:10.1016/S0022-5347(05)65157-9

[9] H. W. Herr and P. C. Sogani, "Does Early Cystectomy Improve the Survival of Patients with High Risk Superficial Bladder Tumors?” Journal of Urology, Vol. 166, No. 4, 2001, pp. 1296-1299. doi:10.1016/S0022-5347(05)65756-4

[10] T. J. Guzzo, A. Magheli, T. J. Bivalacqua, M. E. Nielsen, F. J. Attenello, M. P. Schoenberg and M. L. Gonzalgo, "Pathological Upstaging during Radical Cystectomy Is Associated with Worse Recurrence-Free Survival in Patients with Bacillus Calmette-Guerin-Refractory Bladder Cancer," Urology, Vol. 74, No. 6, 2009, pp. 1276-1280. doi:10.1016/j.urology.2009.05.092

[11] M. Brake, H. Loertzer, R. Horsch and H. Keller, "Recurrence and Progression of Stage T1, Grade 3 Transitional Cell Carcinoma of the Bladder Following Intravesical Immunotherapy with Bacillus Calmette-Guerin," Journal of Urology, Vol. 163, No. 6, 2000, pp. 1697-1701. doi:10.1016/S0022-5347(05)67523-4

[12] L. Cheng, A. L. Weaver, R. M. Neumann, B. G. Scherer 
and D. G. Bostwick, "Substaging of T1 Bladder Carcinoma Based on the Depth of Invasion as Measured by Micrometer: A New Proposal," Cancer, Vol. 86, No. 6, 1999, pp. 1035-1043.

doi:10.1002/(SICI)1097-0142(19990915)86:6<1035::AID -CNCR20>3.0.CO;2-D

[13] L. Cheng, R. M. Neumann, A. L. Weaver, B. E. Spotts and D. G. Bostwick, "Predicting Cancer Progression in Patients with Stage T1 Bladder Carcinoma," Journal of Clinical Oncology, Vol. 17, No. 10, 1999, pp. 3182-3187.

[14] R. Montironi, R. Mazzucchelli, M. Scarpelli, A. LopezBeltran and L. Cheng, "Morphological Diagnosis of Urothelial Neoplasms," Journal of Clinical Pathology, Vol. 61, No. 1, 2008, pp. 3-10. doi:10.1136/jcp.2007.049312

[15] M. Younes, J. Sussman and L. D. True, "The Usefulness of the Level of the Muscularis Mucosae in the Staging of Invasive Transitional Cell Carcinoma of the Urinary Bladder," Cancer, Vol. 66, No. 3, 1990, pp. 543-548. doi:10.1002/1097-0142(19900801)66:3<543::AID-CNCR 2820660323>3.0.CO;2-R

[16] J. C. Angulo, J. I. Lopez, D. J. Grignon and M. SanchezChapado, "Muscularis Mucosa Differentiates Two Populations with Different Prognosis in Stage T1 Bladder Cancer," Urology, Vol. 45, No. 1, 1995, pp. 47-53. doi:10.1016/S0090-4295(95)96490-8

[17] S. Holmäng, H. Hedelin, C. Anderström, E. Holmberg and S. L. Johansson, "The Importance of the Depth of Invasion in Stage T1 Bladder Carcinoma: A Prospective Cohort Study," Journal of Urology, Vol. 157, No. 3, 1997, pp. 800-803. doi:10.1016/S0022-5347(01)65044-4

[18] G. G. Hermann, T. Horn and K. Steven, "The Influence of the Level of Lamina Propria Invasion and the Prevalence of p53 Nuclear Accumulation on Survival in Stage T1 Transitional Cell Bladder Cancer," Journal of Urology, Vol. 159, No. 1, 1998, pp. 91-94. doi:10.1016/S0022-5347(01)64021-7

[19] R. E. Jimenez, E. Gheiler, P. Oskanian, R. Tiguert, W. Sakr, D. P. Wood Jr., J. E. Pontes and D. J. Grignon, "Grading the Invasive Component of Urothelial Carcinoma of the Bladder and Its Relationship with Progression-Free Survival," The American Journal of Surgical Pathology, Vol. 24, No. 7, 2000, pp. 980-987. doi:10.1097/00000478-200007000-00009

[20] N. Edgerton, S. J. Sirintrapun, M. Munoz, Z. Chen and A. O. Osunkoya, "Micropapillary Urothelial Carcinoma of the Urinary Bladder: A Clinicopathological Analysis of 24 Cases," International Journal of Urology, Vol. 18, No. 1, 2011, pp. 49-54. doi:10.1111/j.1442-2042.2010.02672.x

[21] M. Kamat, J. R. Gee, C. P. Dinney, H. B. Grossman, D. A. Swanson, R. E. Millikan, M. A. Detry, T. L. Robinson and L. L. Pisters, "The Case for Early Cystectomy in the Treatment of Nonmuscle Invasive Micropapillary Bladder Carcinoma," Journal of Urology, Vol. 175, No. 3, 2006, pp. 881-885. doi:10.1016/S0022-5347(05)00423-4

[22] M. Kamat, C. P. Dinney, J. R. Gee, H. B. Grossman, A. O. Siefker-Radtke, P. Tamboli, M. A. Detry, T. L. Robinson and L. L. Pisters, "Micropapillary Bladder Cancer: A Review of the University of Texas M.D. Anderson Cancer Center Experience with 100 Consecutive Patients," Cancer, Vol. 110, No. 1, 2007, pp. 62-67. doi:10.1002/cncr.22756 